Signal \& Image Processing : An International Journal (SIPIJ) Vol.5, No.1, February 2014

\title{
ROBUST CONTENT BASED WATERMARKING ALGORITHM USING SINGULAR VALUE DECOMPOSITION OF RADIAL SYMMETRY MAPS
}

\author{
Lakehal Elkhamssa $^{1}$ and Benmohammed Mohamed $^{2}$ \\ ${ }^{1}$ LAMIE laboratory, Department of Computer Engineering, \\ Batna University, Algeria \\ ${ }^{2}$ LIRE laboratory, Department of Computer Engineering, \\ Constantine University, Algeria
}

\begin{abstract}
Nowadays, image content is frequently subject to different malicious manipulations. To protect images from this illegal manipulations computer science community have recourse to watermarking techniques. To protect digital multimedia content we need just to embed an invisible watermark into images which facilitate the detection of different manipulations, duplication, illegitimate distributions of these images. In this work a robust watermarking technique is presented that embedding invisible watermarks into colour images the singular value decomposition bloc by bloc of a robust transform of images that is the Radial symmetry transform. Each bit of the watermark is inserted in a bloc of eight pixels large of the blue channel a high singular value of the corresponding bloc into the radial symmetry map. We justified the insertion in the blue channel by our feeble sensibility to perturbations in this colour channel of images. We present also results obtained with different tests. We had tested the imperceptibility of the mark using this approach and also its robustness face to several attacks.
\end{abstract}

\section{KEYWORDS}

Robust image watermarking, Invisible image watermarking, Radial symmetry maps, Singular value decomposition

\section{INTRODUCTION}

In recent decades, images are widely used, due to the progressive development of new imaging techniques and devices. Users of these techniques and devices share generally images over communication channels that can be unsecure. This wide transmission of image content make it easily modified and generally disregarding author's ownership that is gradually in danger because of these illegitimate manipulations. So the multimedia content protection became a rigorous need to defend the author's copyright and to protect multimedia content from different illegal manipulations. 
Signal \& Image Processing : An International Journal (SIPIJ) Vol.5, No.1, February 2014

As a response to this need of protection come the digital watermarking of multimedia contents [1], [2]; which is no other than information hidden in the multimedia content such that a slight modification on the content results in a modification of the information hidden which is considered as a sign of unauthorized content's manipulation. A survey of watermarking techniques can be found in [3].

\section{RELATED WORK}

Our watermarking method belongs to the second generation approaches. In such approaches image content is used in digital watermarking throw the insertion of the mark in specific points of image like points of interest, edges, corners or other features [4]. A feature-based watermarking scheme was first proposed in [5] where the authors use the Mexican Hat wavelet scale interaction to extract features in the image that can resist a series of attacks which makes them suitable to be used as emplacement to insert and to extract the mark. Another content-based approach in [6] uses a reworked copy of the traditional Harris corner detector. The reworked copy calculates the corner response function within a circular window originate from the image centre and covers the largest area of it to resist image centre based rotation attacks. The new detector gives geometrically significant points that can detect possible geometric attacks. We find in [7] the use of interest points from the Harris corner detector to watermark synchronization before the extraction to recover the watermark positions that can be changed during a geometrical attack. To do, authors generate points of interest using the Harris detector after scale normalization in order to get the most stable points in the image by avoiding the sensitive character of the Harris detector face to scale changes. Then they search within a circular region around the detected points whether the detector response of a selected point reaches a local maximum or not. If it is the case they consider the point, otherwise they neglect it. After that they profit from the characteristic of Pseudo Zernike Moments magnitude which is invariant to rotation to design the watermark to embed in a rotation invariant pattern.

Another work [8] uses the robustness of interest's points to select the positions of the insertion which strengthen the relationship between the watermark and the image content. The detection of interest's points makes possible the creation of triangular partitions of the image and afterward the insertion of the watermark in each triangle. We note that image content is also used in watermarking systems in order to resist other geometric attacks like scale changing and translation in [9].

The watermarking scheme proposed in this paper uses image content through singular value decomposition (SVD). The use of SVD in watermarking schemes is not recent; we find in literature many watermarking schemes based on SVD decomposition [10], [11]. The SVD decomposition may be used in spatial or frequency domain. In frequency domain, the SVD decomposition can be combined with lifting wavelet transform like in [12]; it is also possible to combine it with the discrete cosines transform (DCT) as in [13] or many other transformations. The contribution of this work is the use of an SVD decomposition of a strong content transform (radial symmetry map) to insert in the blue channel where the red and the green channels are just used to synchronize the detection of the watermark. 
Signal \& Image Processing : An International Journal (SIPIJ) Vol.5, No.1, February 2014

\section{SCHEME OVERVIEW}

\subsection{Imperceptibility and Robustness}

A watermark can be visible or invisible, robust or fragile however in authors ownership protection applications a good watermarking algorithm is the one which is invisible and robust to different attacks. So in our proposed algorithm we take into account these two metrics to achieve a successful algorithm for the author copyright protection applications. To be invisible to human visual eye, first, the mark is inserted in the blue channel, which is the one to which the human visual system is less sensitive [14]. Second, the watermark bits are inserted in points of high interest in the image; those points with high luminance values make the modifications in the image imperceptible. That is the property of imperceptibility which mast marks any consistent watermarking scheme; the other property is the robustness of the system [15]. The robustness of our scheme is achieved by using robust transform of image information. This transform is called radial symmetry transform and it presents very good robustness against image transformations and nosing. The radial symmetry transform is originally used to detect image features [16], [17] and we use it in this work to support the robustness of the scheme and to detect pixels which care the mark bits.

\subsection{Radial Symmetry Maps}

Radial symmetry maps are generally used to detect interest points in images which correspond to object's centres. We find in literature many works on radial symmetry maps but the most commonly used is the one of [18]. Author in [18] details an algorithm to calculate the radial symmetry map of an image. With this approach a symmetry score is calculated from votes of one pixel to surrounding pixels. The transform is calculated in one or more radii $n$. the value of the transform at radius $n$ indicates the contribution to radial symmetry of the gradients a distance $n$ away from each point.

At each radius $n$, an orientation projection image $O_{n}$ and a magnitude projection image $M_{n}$ are formed. These images are generated by examining the gradient $g$ at each point $p$ from which a corresponding positively-affected pixel $\mathrm{p}_{+\mathrm{ve}}(\mathrm{p})$ and negatively-affected pixel $\mathrm{p}_{\text {-ve }}(\mathrm{p})$ are determined, as shown in Figure 1. The positively-affected pixel is defined as the pixel that the gradient vector $\mathrm{g}(\mathrm{p})$ is pointing to, a distance $\mathrm{n}$ away from $\mathrm{p}$, and the negatively-affected pixel is the pixel a distance $n$ away that the gradient is pointing directly away from.

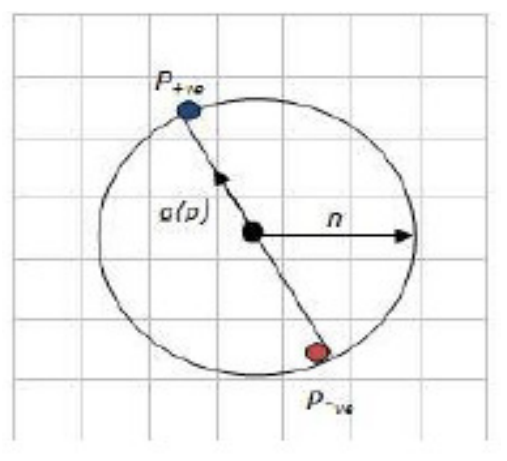

Figure 1. Positively and negativelly affected pixels 
Coordinates of the positively-affected pixel are given by:

$$
p_{+v e}=p+\operatorname{ROUND}\left(n \cdot \frac{g(p)}{\|g(p)\|}\right)
$$

Coordinates of the negatively-affected pixel are given by:

$$
p_{-v e}=p-\operatorname{ROUND}\left(n \cdot \frac{g(p)}{\|g(p)\|}\right)
$$

The orientation and magnitude projection images are initially zero. For each pair of affected pixels, the corresponding point $\mathrm{p}_{+\mathrm{v}}$ in the orientation projection image $\mathrm{O}_{\mathrm{n}}$ and magnitude projection image $M_{n}$ is incremented by 1 and $\|g(p)\|$, respectively, while the point corresponding to $\mathrm{p}_{\text {-ve }}$ is decremented by these same quantities in each projection image. That is:

$$
\begin{aligned}
& O_{n}\left(p_{+v e}(p)\right)=O_{n}\left(p_{+v e}(p)\right)+1 \\
& O_{n}\left(p_{\text {-ve }}(p)\right)=O_{n}\left(p_{\text {-ve }}(p)\right)-1 \\
& M_{n}\left(p_{+v e}(p)\right)=M_{n}\left(p_{+v e}(p)\right)+\|g(p)\| \\
& M_{n}\left(p_{\text {-ve }}(p)\right)=M_{n}\left(p_{\text {-ve }}(p)\right)-\|g(p)\|
\end{aligned}
$$

The radial symmetry contribution at radius $\mathrm{n}$ is defined as the convolution:

Where:

$$
\begin{gathered}
\mathrm{S}_{\mathrm{n}}=\mathrm{F}_{\mathrm{n}} * \mathrm{~A}_{\mathrm{n}} \\
F_{n}(p)=\frac{M_{n}(p)}{k_{n}}\left(\frac{\left|\tilde{O}_{n}(p)\right|}{k_{n}}\right)^{\alpha}
\end{gathered}
$$

And

$$
\tilde{O}_{n}(p)= \begin{cases}O_{n}(p) & \text { if } \mathrm{On}<\mathrm{kn} \\ k_{n} & \text { else }\end{cases}
$$

$\mathrm{A}_{\mathrm{n}}$ is a two-dimensional Gaussian, $\alpha$ is the radial strictness parameter, and $\mathrm{k}_{\mathrm{n}}$ is a scaling factor that normalizes $\mathrm{M}_{\mathrm{n}}$ and $\mathrm{O}_{\mathrm{n}}$ across different radii.

The full map is defined as the average of the symmetry contributions over all the radii considered: 
Signal \& Image Processing : An International Journal (SIPIJ) Vol.5, No.1, February 2014

$$
S=\frac{1}{|N|} \sum_{n \in N} S_{n}
$$

\section{THE PROPOSED SCHEME}

The proposed algorithm inserts the watermark bits into the blue channel of the image based on an SVD decomposition of this channel. The SVD decomposition used here is a bloc based one which necessitates the decomposition of the blue channel into blocs of 8 pixels large in different locations in the image. The locations are objects centres obtained after a local maxima search over the radial symmetry map.

At the same time we should calculate the radial symmetry map using the red and green channel of the original image. The symmetry map is also divided into blocks of 8 pixels large in the same locations as the blue channel.

\subsection{Insertion of the watermark}

To insert the pixel of the binary mark we need to calculate the $s v d$ of both, the blue block $i$ and the symmetry transform map of the block $i$ as indicated in Figure 2.

The insertion mechanism uses the formula:

$$
\sigma_{b k}=W_{i} * \sigma_{s k} * \alpha
$$

Where $\mathrm{k}$ is the index of the singular value which holds the mark.

$\alpha$ is the embedding strength (an adjustment parameter between the quality and the robustness). It is chosen experimentally.

$\sigma_{\mathrm{bk}}, \sigma_{\mathrm{sk}}$ are respectively singular values from the range of big singular values of the blue bloc and those of the radial symmetry map bloc.

\subsection{Extraction of the watermark}

Figure 3 presents the extraction scheme; it is based on the decomposition of blue image channel and radial symmetry map into blocs, then the decomposition of each bloc to its singular values.

To extract the watermark we use the singular values following the formula:

$$
\widetilde{W} i=\sigma_{b k w} / \sigma_{s k w} / \beta
$$

Where $\beta$ is $1 / 2 \alpha, \sigma_{\mathrm{bkw}}$ and $\sigma_{\mathrm{skw}}$ are respectively singular values from high singular values of the blue blocs and those of the radial symmetry map of the watermarked image. 
Signal \& Image Processing : An International Journal (SIPIJ) Vol.5, No.1, February 2014

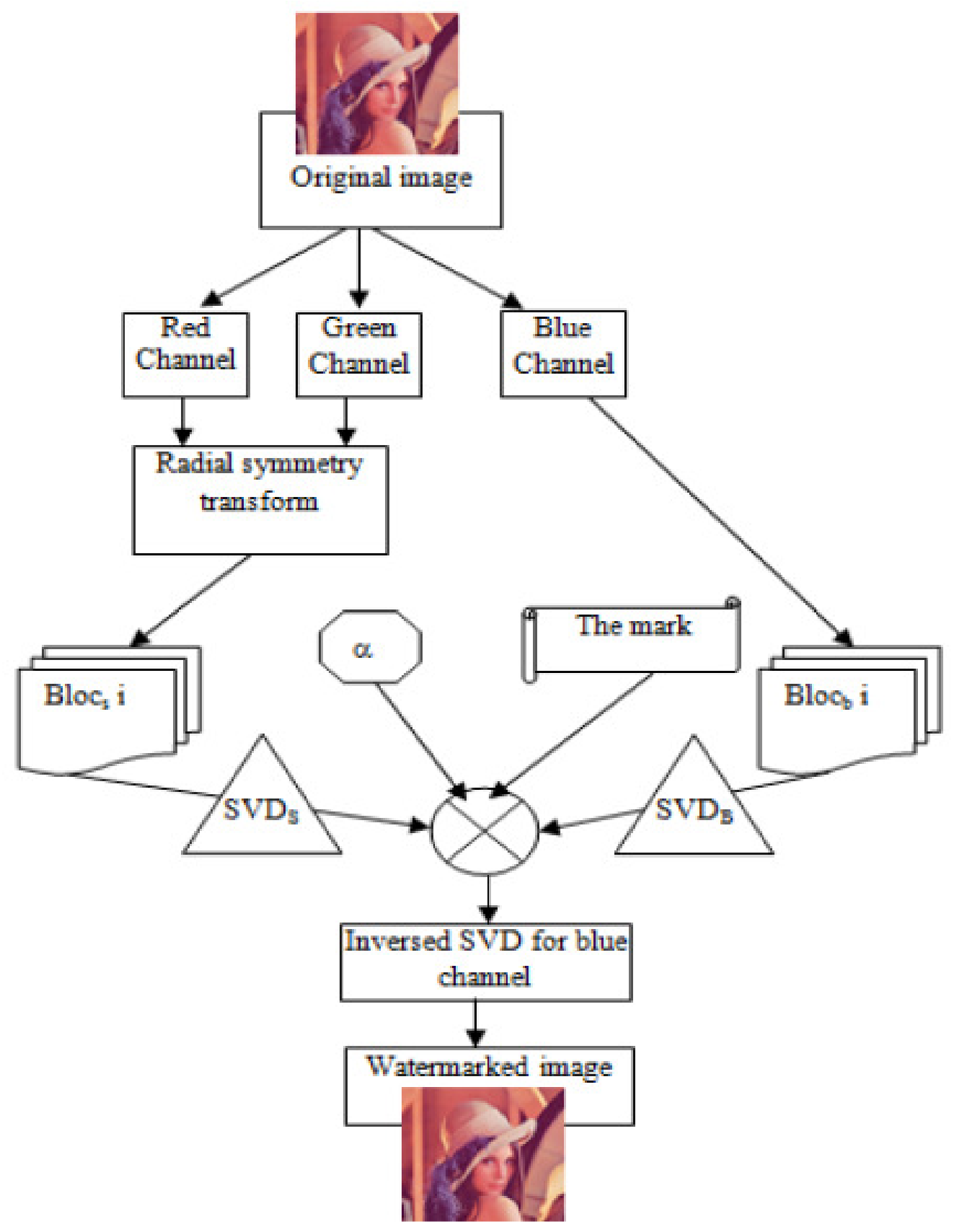

Figure 2. The mark insertion scheme

\section{RESULTS AND DISCUSSION}

This section presents tests results of imperceptibility and robustness for our scheme. We have tested our algorithm with and without attacks; table 1 presents the obtained results without attacks and table 3 and table 4 present the results in presence of attacks. To test our watermarking scheme we use an image database composed of color images of size 512 x 512 pixels: Lena, baboon, air plane, peppers. The mark used is a binary image of size $8 \times 8$ pixels. 
Signal \& Image Processing : An International Journal (SIPIJ) Vol.5, No.1, February 2014

Figure 4 presents the original images used in tests with the watermark image to the right bottom of the figure.

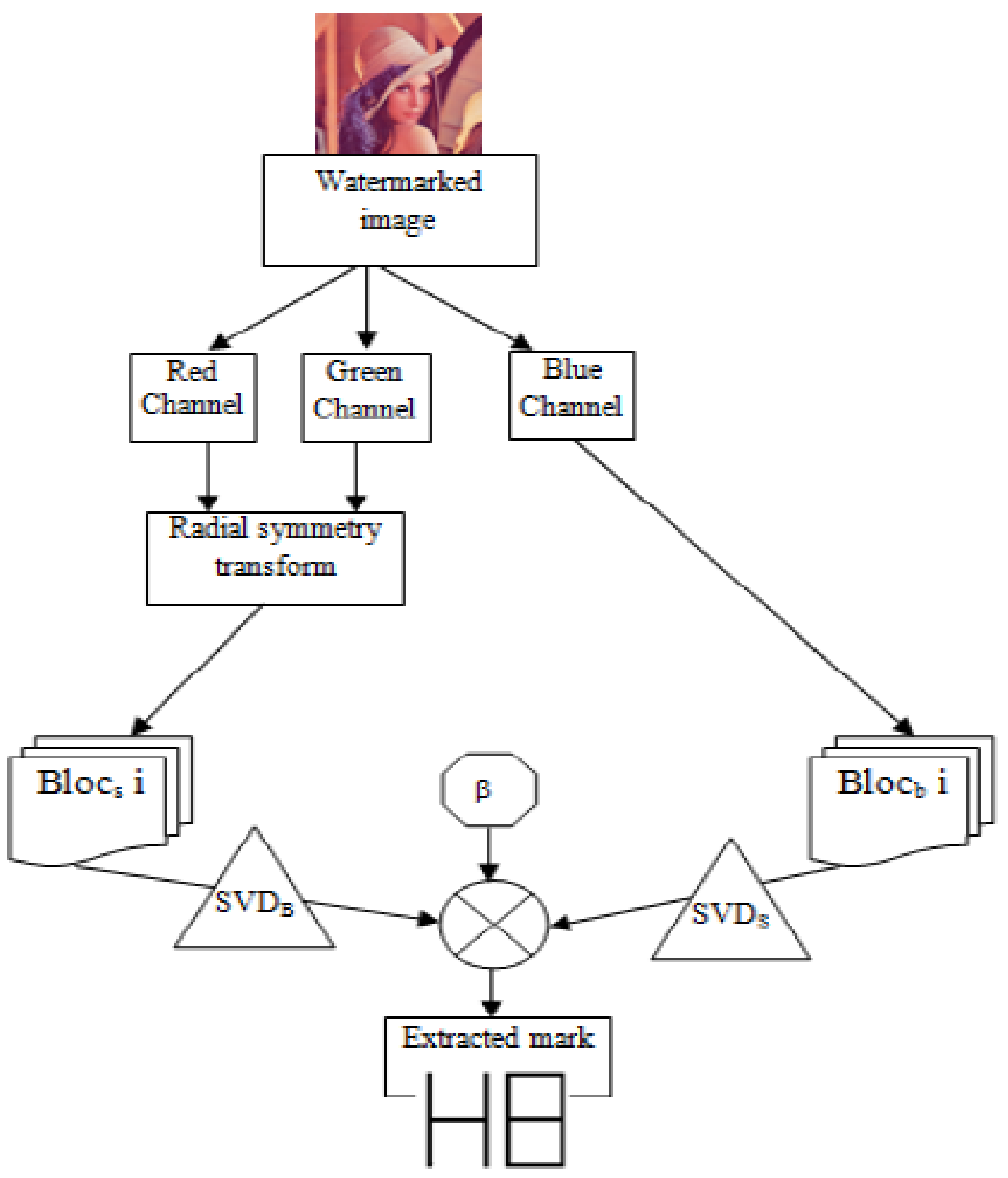

Figure 3. The mark extraction scheme 
Signal \& Image Processing : An International Journal (SIPIJ) Vol.5, No.1, February 2014
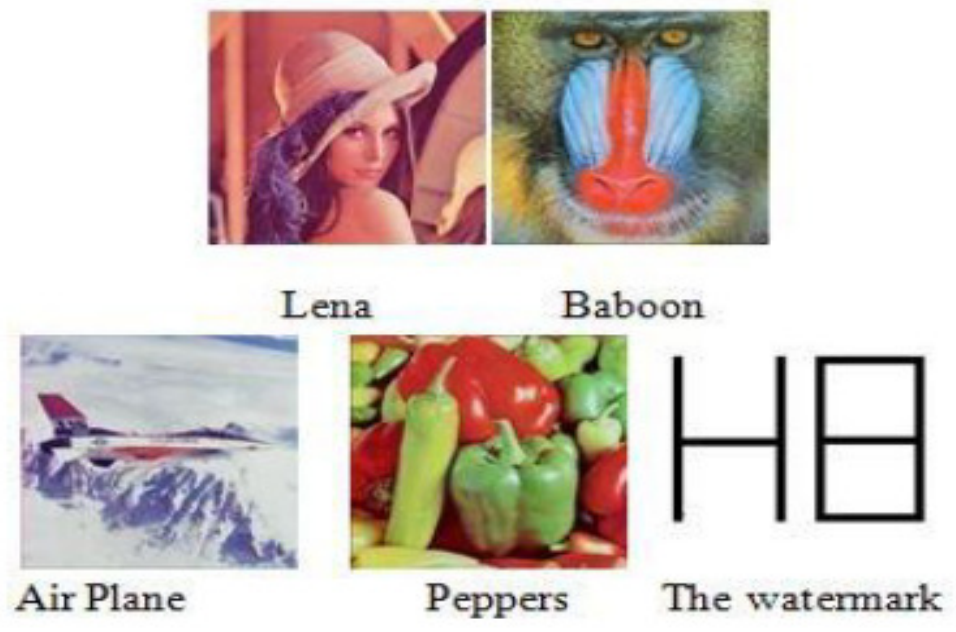

Figure 4. Test images with the watermark used

The table 1 presents the imperceptibility results according to tree objective measures: PSNR (Peak Signal to Noise Ratio) calculated between the original image and the watermarked one, NC (Normal Correlation) and BCR (Bit Correct Ratio) calculated between the original watermark and the extracted one.

We note that the results obtained with the PSNR measure between original image and the watermarked one are very good when we know that authors considered a PSNR rate as good when it is greater than $30 \mathrm{~dB}$. With tested images we have PSNR from 42 to $46 \mathrm{~dB}$.

When investigating table 1 we find that the $\mathrm{NC}$ measure is between 0.97 and 1 when the BCR measure is between $98.44 \%$ and $100 \%$. These are very interesting rates which indicates that the proposed algorithm makes a high-quality extraction of the watermark.

To evaluate the robustness of our algorithm we should test its performance face to different attacks. The attack chosen are common in watermarking algorithm evaluation and includes low pass filtering, noise, jpeg compression and cropping. In this paper, we have used attacks with different parameters to check as deeply as possible the robustness of the proposed algorithm. Attacks used to test our watermarking system are given in table 2.

In this paper we have not tested the capacity of our proposed scheme, but we claim that our algorithm has a very good capacity since it inserts watermark bits into radial symmetry transform local maxima. 
Signal \& Image Processing : An International Journal (SIPIJ) Vol.5, No.1, February 2014

Table 1. imperceptibility and robustness of the watermark in absence of attacks.

\begin{tabular}{|c|c|c|c|}
\hline Image & PSNR & NC & BCR $\%$ \\
\hline Lena & $+46.13 \mathrm{~dB}$ & 1 & 100 \\
\hline Baboon & $+42.76 \mathrm{~dB}$ & 0.97 & 98.44 \\
\hline Air Plane & $+45.42 \mathrm{~dB}$ & 0.97 & 98.44 \\
\hline Peppers & $+45.42 \mathrm{~dB}$ & 1 & 100 \\
\hline
\end{tabular}

Table 2. Attacks used in different tests.

\begin{tabular}{|c|c|c|}
\hline & Attacks & Parameters \\
\hline SP1 & Salt \& Pepper noise & Density 0.008 \\
\hline SP2 & Salt \& Pepper noise & Density 0.002 \\
\hline SP3 & Salt \& Pepper noise & Density 0.05 \\
\hline GN1 & Gaussian noise & $\mathrm{M}=0.0 \mathrm{~V}=0.001$ \\
\hline GN2 & Gaussian noise & $\mathrm{M}=0.1 \mathrm{~V}=0.001$ \\
\hline GF & Gaussian filter & $3 \times 3$ \\
\hline SH & Sharpening & $3 \times 3$ \\
\hline HE & Histogram equalization & \\
\hline MF & Median filter & $3 \times 3$ \\
\hline JC1 & JPEG Compression & Quality 80 \\
\hline JC2 & JPEG Compression & Quality 60 \\
\hline CR & Cropping & $1 / 8$ of image \\
\hline AF & Average filter & $3 \times 3$ \\
\hline LF & Laplacian filter & $3 \times 3$ \\
\hline Rot1 & Rotation & $0.2^{\circ}$ \\
\hline
\end{tabular}

Table 3 presents the NC and BCR measure between the watermark inserted and the one extracted using our extraction scheme with different attacks over four test images.

By observing the interval of these two objective measures over all attacks (NC between 0.62 and 1, BCR between $71.88 \%$ and $100 \%$ ) we can deduce that the proposed algorithm perform well face to these attacks. Furthermore, the robustness of the method is related to the tested image, thus to the content. Then, enhancing the content transform may enhance the extraction

The robustness of the algorithm face to the rotation attack can be reinforced using a robust rotational radial symmetry transform. 
Signal \& Image Processing : An International Journal (SIPIJ) Vol.5, No.1, February 2014

In the next figures (5-9) we present comparison graphics of NC rates between watermarks extracted with our algorithm and those presented in reference [12].

Algorithm in [12] uses gray scale images of $256 \times 256$ pixels where the watermark image is a binary image of $32 \times 32$ pixels which represents the letter "A". Table 4 summarises results of comparison of our algorithm and the algorithm of [12]. All algorithm [12] robustness values are extracted from reference [12].

Table 3. Robustness of the proposed scheme (NC and BCR) over different attacks

\begin{tabular}{|c|c|c|c|c|c|c|c|c|}
\hline $\mathbf{N}^{\circ}$ & \multicolumn{2}{|c|}{ Lena } & \multicolumn{2}{c|}{ Baboon } & \multicolumn{2}{c|}{ Air plane } & \multicolumn{2}{c|}{ Peppers } \\
\hline & NC & BCR\% & NC & BCR\% & NC & BCR\% & NC & BCR\% \\
\hline SP1 & 0.88 & 89.06 & 0.97 & 98.44 & 1.00 & 100 & 1.00 & 100 \\
\hline SP2 & 1.00 & 100 & 0.97 & 98.44 & 0.97 & 98.44 & 1.00 & 100 \\
\hline SP3 & 0.73 & 71.88 & 0.90 & 78.13 & 0.90 & 79.69 & 0.73 & 75.00 \\
\hline GN1 & 1.00 & 100 & 0.97 & 98.44 & 1.00 & 100 & 0.97 & 98.44 \\
\hline GN2 & 1.00 & 100 & 0.97 & 96.88 & 0.97 & 98.44 & 0.97 & 98.44 \\
\hline GF & 1.00 & 100 & 0.97 & 98.44 & 0.97 & 98.44 & 1.00 & 100 \\
\hline SH & 1.00 & 93.75 & 0.97 & 98.44 & 1.00 & 100 & 0.97 & 98.44 \\
\hline HE & 1.00 & 100 & 0.97 & 98.44 & 0.91 & 95.31 & 0.84 & 92.19 \\
\hline MF & 0.90 & 95.31 & 0.82 & 90.63 & 0.77 & 87.50 & 0.71 & 82.81 \\
\hline JC1 & 1.00 & 100 & 0.93 & 96.88 & 1.00 & 100 & 0.84 & 92.19 \\
\hline JC2 & 0.81 & 90.63 & 0.73 & 87.50 & 0.94 & 96.88 & 0.63 & 81.25 \\
\hline CR & 0.93 & 95.31 & 0.98 & 98.44 & 0.93 & 95.31 & 1.00 & 100 \\
\hline AF & 0.85 & 92.19 & 0.73 & 84.38 & 0.66 & 79.69 & 0.62 & 78.13 \\
\hline LF & 0.71 & 85.94 & 0.90 & 95.31 & 0.90 & 95.31 & 0.80 & 90.63 \\
\hline Rot1 & 0.90 & 93.31 & 0.84 & 92.19 & 0.85 & 92.19 & 0.81 & 90.63 \\
\hline
\end{tabular}

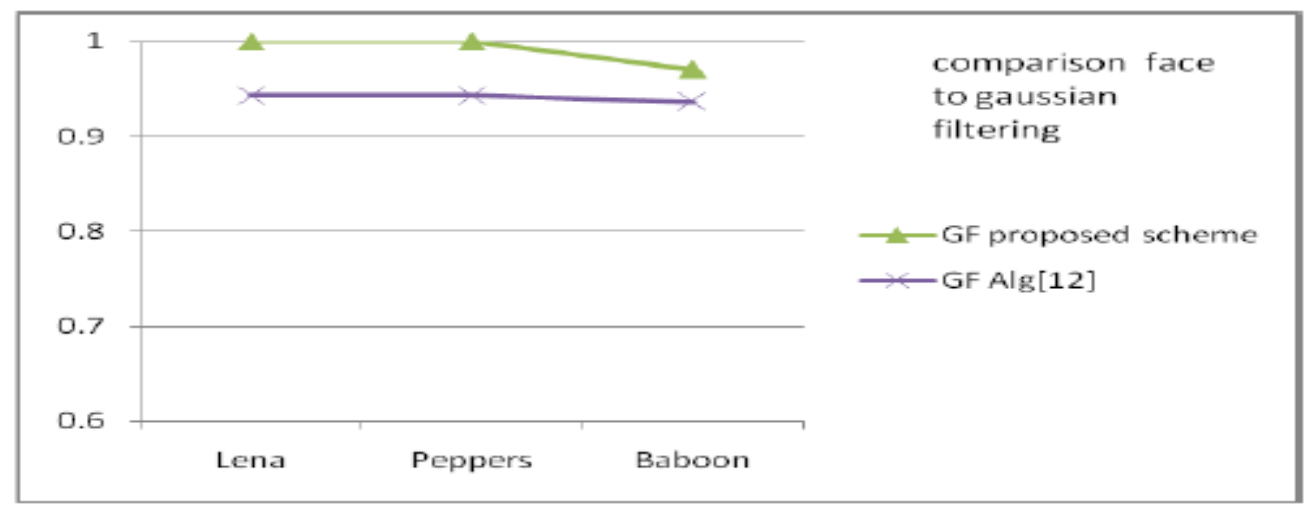

Figure 5. Comparaison of $\mathrm{NC}$ rates face to gaussian filtering 
Signal \& Image Processing : An International Journal (SIPIJ) Vol.5, No.1, February 2014

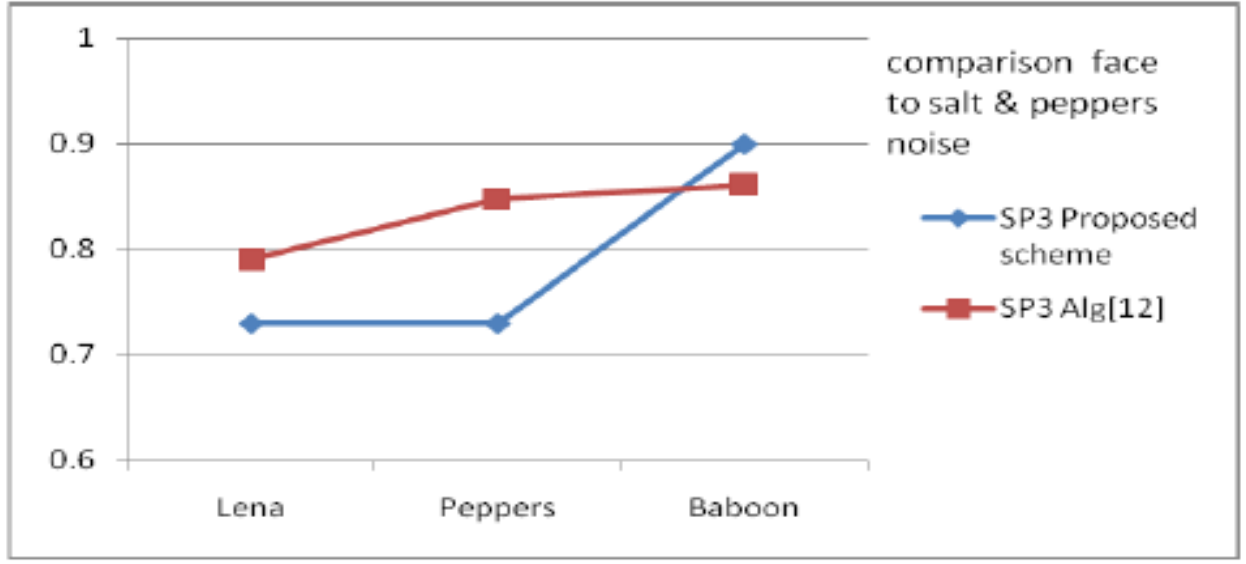

Figure 6. Comparaison of $\mathrm{NC}$ rates face to salt \& peppers noise (density 0.05 )

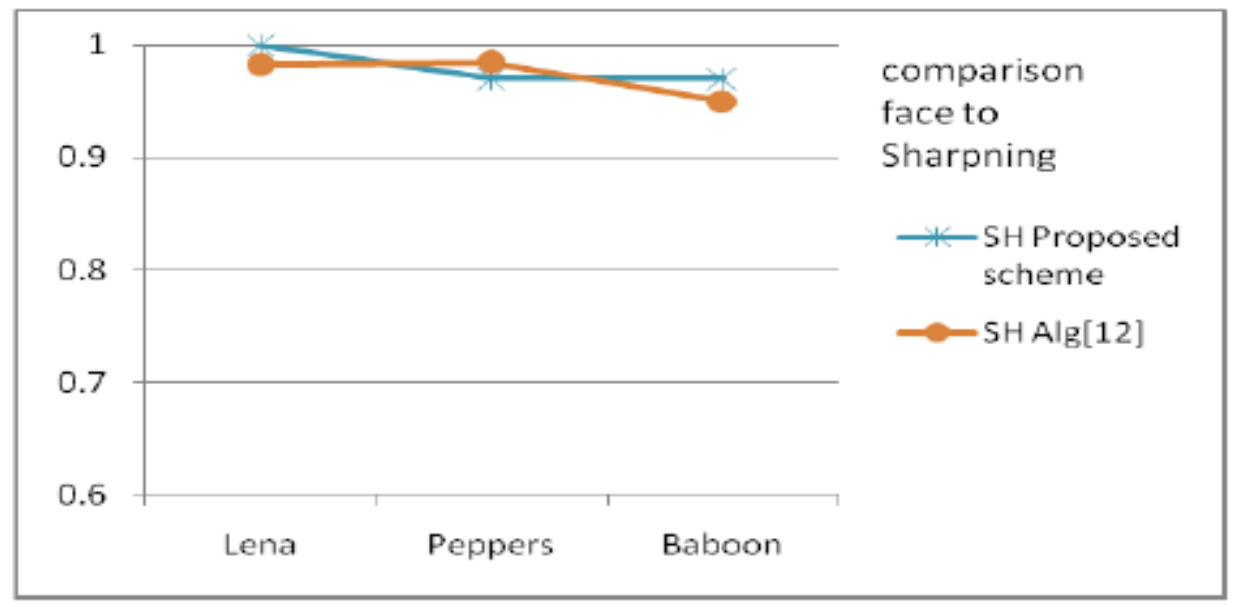

Figure 7. Comparaison of $\mathrm{NC}$ rates face to sharpening attack

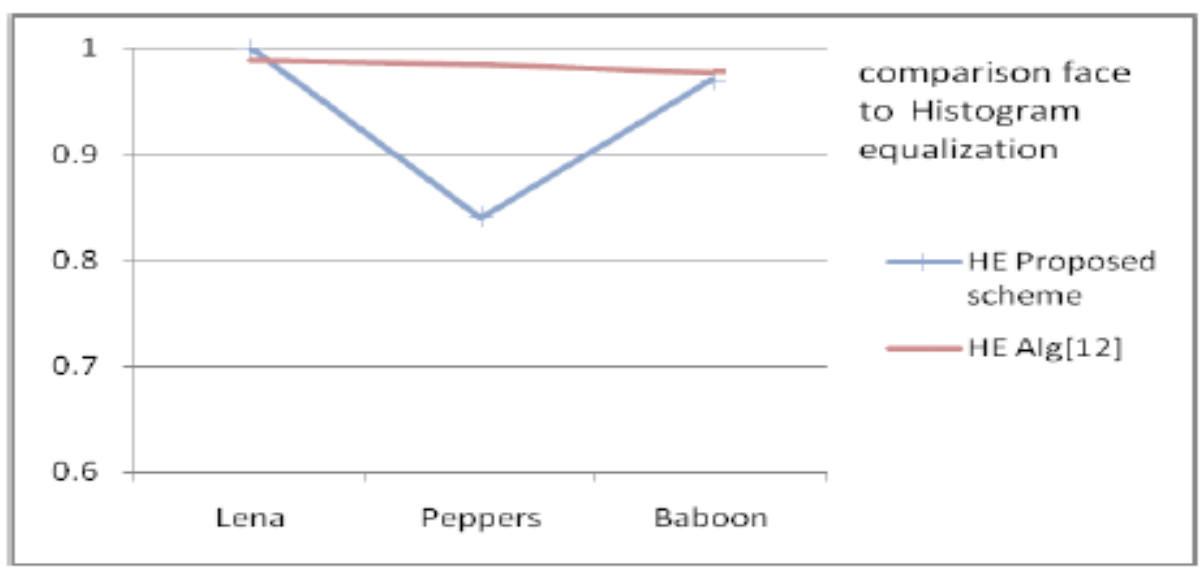

Figure 8. Comparaison $\mathrm{NC}$ rates face to histogram equalization attack 
Signal \& Image Processing : An International Journal (SIPIJ) Vol.5, No.1, February 2014

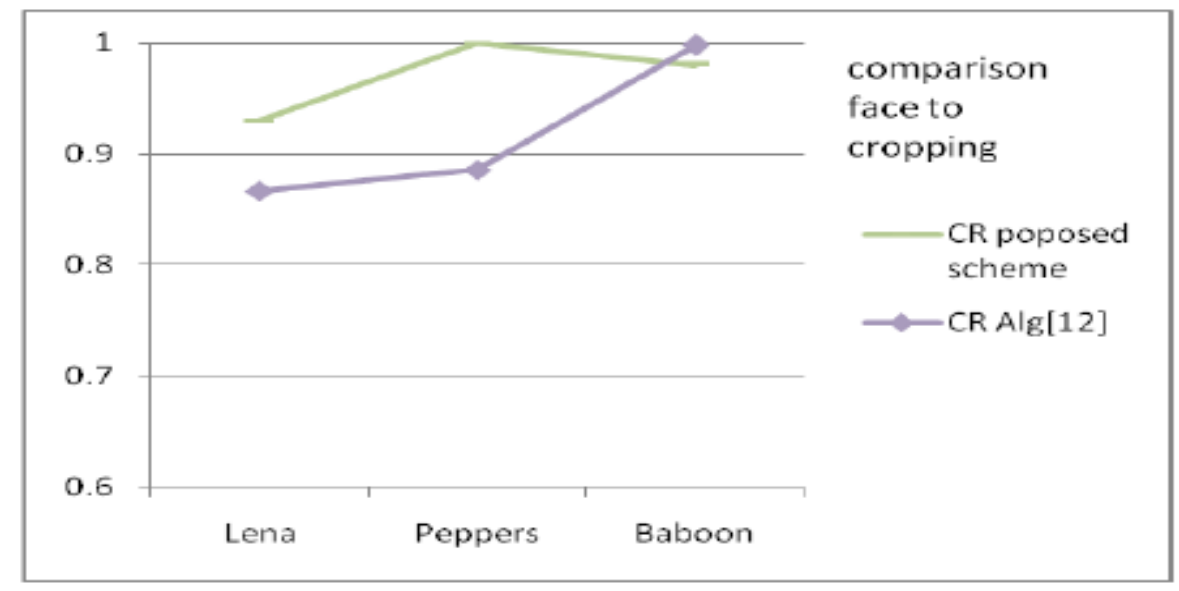

Figure 9. Comparaison of $\mathrm{NC}$ rates face to cropping attack

Table 4. Comparaison of the proposed algorithm with the algorithm of [12] in term of the two metrics NC and BCR

\begin{tabular}{|c|c|c|c|c|c|c|c|c|c|c|c|c|}
\hline & \multicolumn{4}{|c|}{ Lena } & \multicolumn{4}{|c|}{ Peppers } & \multicolumn{4}{|c|}{ Baboon } \\
\hline$\frac{\mathscr{v}}{\underline{y}}$ & $\begin{array}{r}\text { Prop } \\
\text { sch }\end{array}$ & $\begin{array}{l}\text { posed } \\
\text { leme }\end{array}$ & Alg & {$[12]$} & $\begin{array}{r}\text { Prop } \\
\text { sch }\end{array}$ & & Alg & {$[12]$} & & $\begin{array}{l}\text { posed } \\
\text { leme }\end{array}$ & Alg & $\mathrm{g}[12]$ \\
\hline $\mathrm{N}^{\circ}$ & $\mathrm{NC}$ & BCR\% & $\mathrm{NC}$ & BCR\% & $\mathrm{NC}$ & BCR\% & $\mathrm{NC}$ & $\mathrm{BCR} \%$ & $\mathrm{NC}$ & BCR\% & $\mathrm{NC}$ & BCR\% \\
\hline SP1 & 0.88 & 89.06 & - & - & 1.00 & 100 & - & - & 0.97 & 98.44 & - & - \\
\hline SP2 & 1.00 & 100 & - & - & 1.00 & 100 & - & - & 0.97 & & - & - \\
\hline SP3 & 0.73 & 71.88 & \begin{tabular}{|l|}
0.790 \\
\end{tabular} & 68.46 & 0.73 & 75.00 & 0.848 & 76.07 & 0.90 & & 0.861 & 78.52 \\
\hline GN1 & 1.00 & & - & - & 0.97 & 98.44 & - & - & 0.97 & & - & - \\
\hline GN2 & 1.00 & 100 & - & - & 0.97 & 98.44 & - & - & 0.97 & 96.88 & - & - \\
\hline GF & 1.00 & 100 & 0.943 & 90.63 & 1.00 & 100 & 0.943 & 90.72 & 0.97 & 98.44 & 0.936 & 89.65 \\
\hline SH & 1.00 & 93.75 & 0.982 & 97.07 & 0.97 & 98.44 & 0.984 & 97.36 & 0.97 & 98.44 & 0.950 & 96.19 \\
\hline $\mathrm{HE}$ & 1.00 & 100 & 0.988 & 98.05 & 0.84 & 92.19 & 0.984 & 97.36 & 0.97 & 98.44 & 0.977 & 96.19 \\
\hline MF & 0.90 & 95.31 & - & - & 0.71 & 82.81 & - & - & 0.82 & 90.63 & - & - \\
\hline $\mathrm{JC} 1$ & 1.00 & 100 & - & - & 0.84 & 92.19 & - & - & 0.93 & 96.88 & - & - \\
\hline $\mathrm{JC} 2$ & 0.81 & 90.63 & - & - & 0.63 & 81.25 & - & - & 0.73 & 87.50 & - & - \\
\hline $\mathrm{CR}$ & 0.93 & 95.31 & \begin{tabular}{|l}
0.867 \\
\end{tabular} & 79.10 & 1.00 & \begin{tabular}{|l|}
100 \\
\end{tabular} & 0.886 & 82.23 & 0.98 & 98.44 & 0.998 & 99.61 \\
\hline $\mathrm{AF}$ & 0.85 & 92.19 & - & - & 0.62 & 78.13 & - & - & 0.73 & 84.38 & - & - \\
\hline LF & 0.71 & 85.94 & - & - & 0.80 & 90.63 & - & - & 0.90 & 95.31 & - & - \\
\hline Rot1 & 0.90 & 93.31 & - & - & 0.81 & 90.63 & - & - & 0.84 & 92.19 & - & - \\
\hline
\end{tabular}

Figures (10-14) present comparison graphics of BCR rates between watermarks extracted with our algorithm and those presented in reference [12]. 
Signal \& Image Processing : An International Journal (SIPIJ) Vol.5, No.1, February 2014

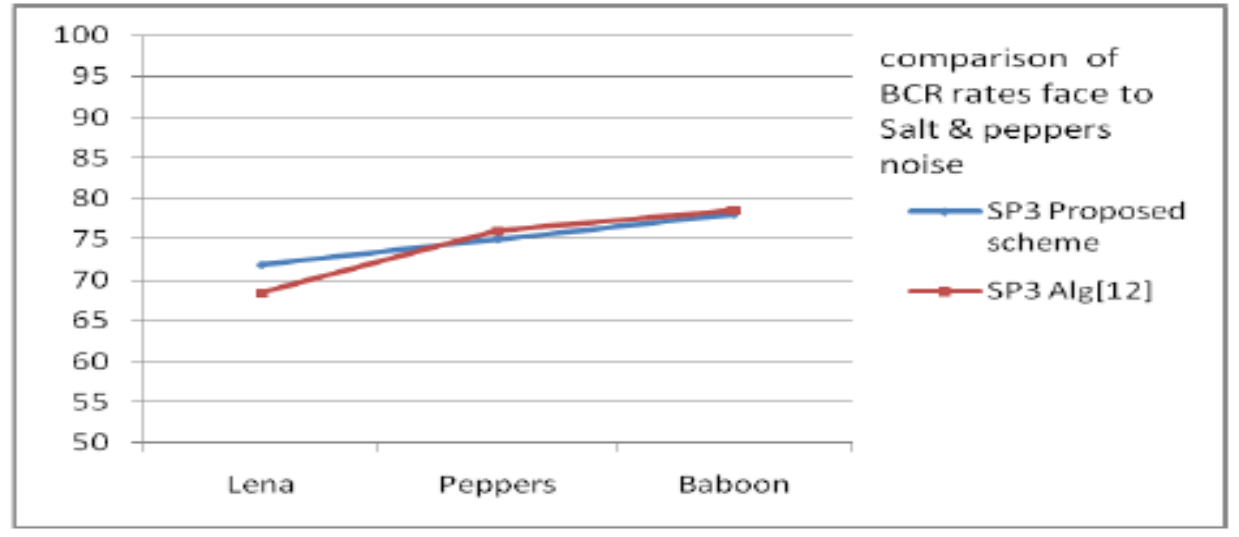

Figure 10. Comparaison of BCR rates face to salt \& peppers noise (density 0.05 )

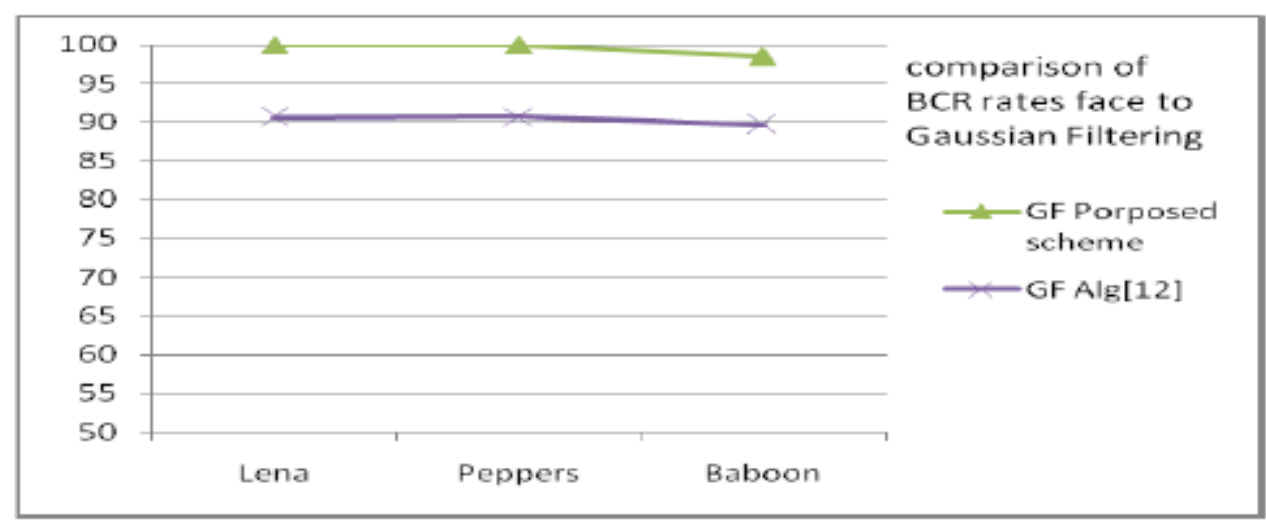

Figure 11. Comparaison of BCR rates face to gaussian filtering

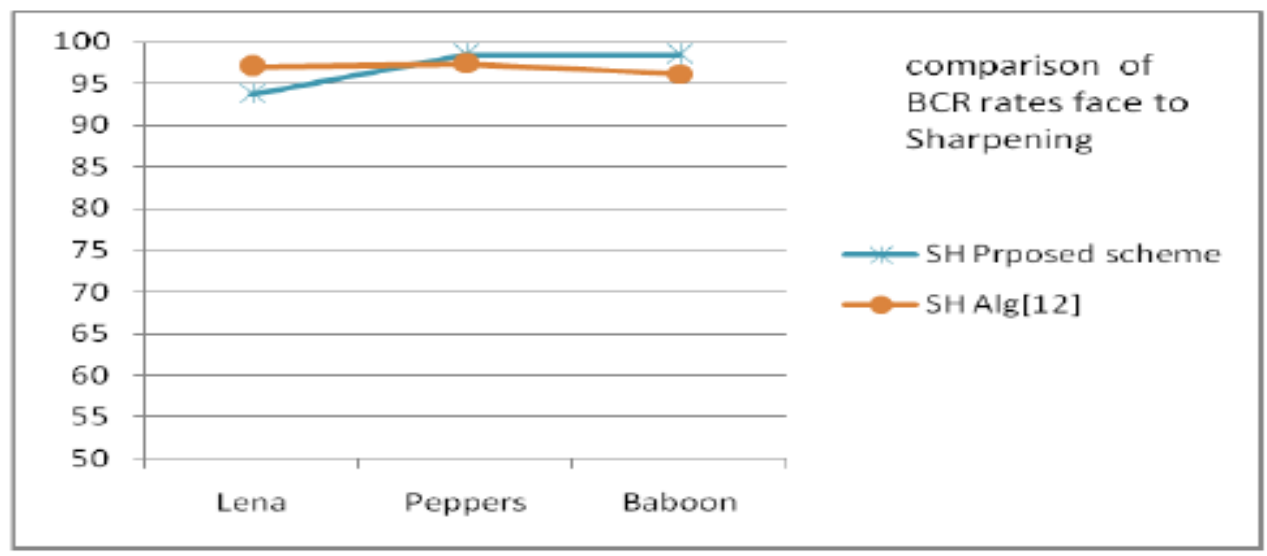

Figure 12. Comparaison of BCR rates face to sharpening attack 
Signal \& Image Processing : An International Journal (SIPIJ) Vol.5, No.1, February 2014

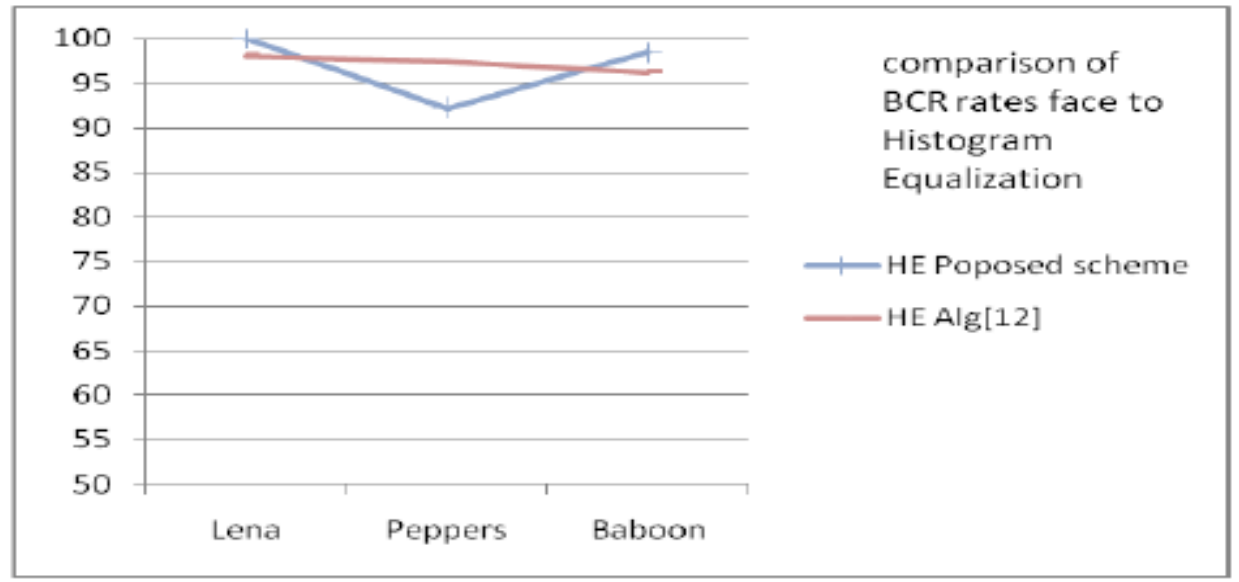

Figure 13. Comparaison BCR rates face to histogram equalization attack

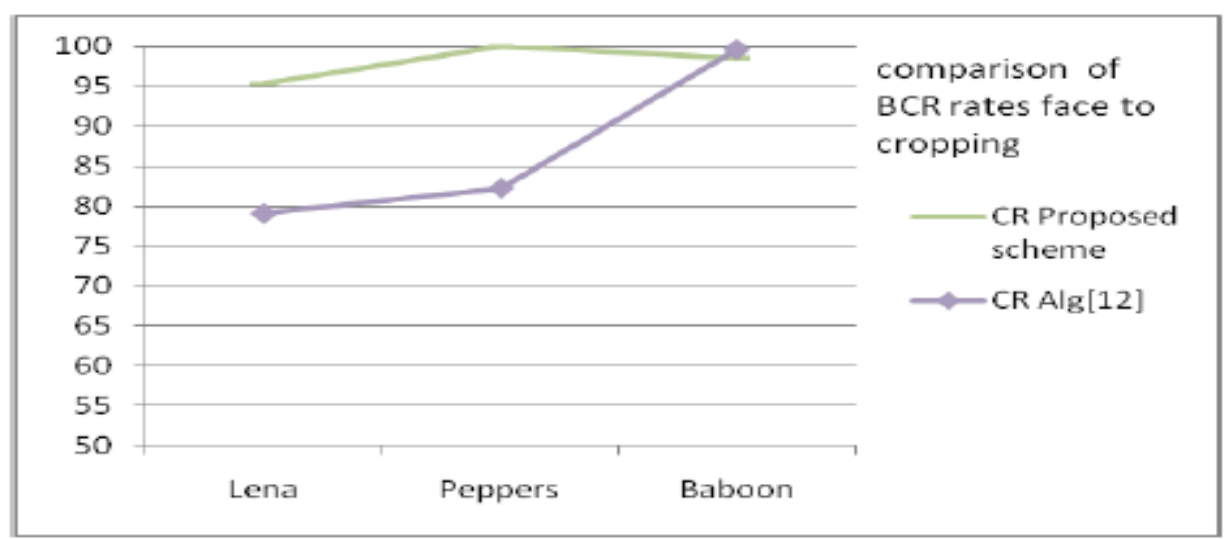

Figure 14. Comparaison of BCR rates face to cropping attack

\section{CONCLUSION AND FURTHER WORKS}

In this work, we present a new content based watermarking scheme which uses image content to improve robustness and two insure the correct extraction of the watermark in author ownership protection applications. The watermarking algorithm presented in this paper is a promised one since it presents good results in terms of robustness and imperceptibility. However, the approach necessitates to be compared to many other works in order to be judged correctly. To this date there are no works which evaluate different content based algorithms face to different attacks using unified metrics. So the comparison of this work with other works should be based on the same attacks and considering the same metrics. Since it is the case, we resolve, in our future work, to evaluate and to compare the present work with other content based watermarking techniques using a unified testing benchmark. We will work also on the enhancement of the radial symmetry maps so the points choosing to care the mark can be the most repetitive ones and the most robust to image manipulations. 
Signal \& Image Processing : An International Journal (SIPIJ) Vol.5, No.1, February 2014

\section{ACKNOWLEDGEMENTS}

Lakehal elkhamssa thanks Pr. Noui L. for his aid and considers his contribution to success this work.

\section{REFERENCES}

[1] I. Cox, M. Miller, and J. Bloom. "Digital Watermarking: Principles \& Practices". Morgan Kaufmann Publisher, San Francisco, USA, 2002.

[2] C. I. Podilchuk and E. J. Delp, "Digital Watermarking: Algorithms and Applications," IEEE Signal Processing Magazine, pp. 33-46, 2001.

[3] V. M. Potdar, S. Han and E. Chang , "A Survey of Digital Image Watermarking Techniques", 3rd international conference on industrial informatics (INDIN), 2005.

[4] M. Kutter, S. K. Bhattacharjee, T. Ebrahimi, "Towards second generation watermarking schemes", international conference on image processing, 1999.

[5] C. W. Tang and H. M. Hang, "A Feature-Based Robust Digital Image Watermarking Scheme", IEEE Transactions on Signal Processing, Vol. 51, pp. 950-959, 2003.

[6] X. Qi and J. Qi, "A robust content-based digital image watermarking scheme," Signal Processing , Vol. 87, pp. 1264-1280, 2007.

[7] L.D. Li, B. L. Guo AND L. Guo, "Combining Interest Point and Invariant Moment for Geometrically Robust Image Watermarking”, Journal of information science and engineering, vol. 25, pp. 499-515, 2009.

[8] P. Bas, J.M. Chassery, B. Macq, "Toward a content-based watermarking scheme”, Journal of signal processing [journal de Traitement du Signal], vol. 19, pp. 11-17, 2002.

[9] D. Simitopoulos, D.E. Koutsonanos, M.G. Strintzis, "Robust Image Watermarking Based on Generalized Radon Transformations", IEEE Trans. on Circuits and Systems for Video Technology, IEEE, vol. 13, pp. 732-745, 2003.

[10] R. Liu and T. Tan, "A svd-based watermarking scheme for protecting right-ful ownership", IEEE Transactions on Multimedia, vol. 4, pp. 121-128, 2002.

[11] C. Bergman, J. Davidson, "Unitary Embedding for Data Hiding with the SVD", Security, Steganography, and Watermarking of Multimedia Contents VII, SPIE vol. 5681, 2005.

[12] K. Loukhaoukha, "Tatouage numérique des images dans le domaine des ondelettes basé sur la décomposition en valeurs singulières et l'optimisation multi-objective", Phd thesis, Laval University , pp. 115-126, 2010.

[13] F. Huang, Z. H. Guan, "A hybrid SVD-DCT watermarking method based on LPSNR", Pattern Recognition Letters,vol. 25, pp. 1769-1775, 2004.

[14] R. W. G. Hunt, The reproduction of color. Fountain press, Tolworth, England, 1988.

[15] F. Hartung and M. Kutter, "Multimedia Watermarking Techniques," Proceedings of the IEEE , vol. 87, pp. 1079-1107, 1999.

[16] G. Heidemann, "Focus of Attention from Local Color Symmetries" IEEE Trans. on Pattern Analysis and Machine Intelligence, vol. 26, pp. 817-830, 2004.

[17] A. Rebai, A. Joly, N. Boujemaa, "Constant Tangential Angle Elected Interest Points," in Proc. of the 8th ACM International Workshop on Multimedia Information Retrieval (MIR), pp. 203-211, 2006.

[18] A. Zelinsky, G. Loy, "Fast Radial Symmetry for Detecting Point of Interest," IEEE Trans. on Pattern Analysis and Machine Intelligence, pp. 959-973, vol. 27, August 2003. 
Signal \& Image Processing : An International Journal (SIPIJ) Vol.5, No.1, February 2014

\section{AUTHOR}

Lakehal elkhamssa is currently a researcher in the LAMIE laboratory, Batna University, Algeria and an assistant professor at the same university. She received her Magister degree in computer science in the University of Batna in 2009. Her main research interests are: image watermarking, image understanding and colour constancy. Actually she prepares her Phd thesis in the University of Batna.

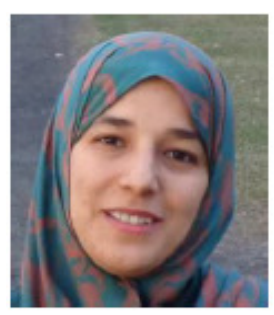

